\title{
The Impact of the Environment on the Early Stages of Radio Source Evolution
}

\author{
Małgosia Sobolewska ${ }^{1}$, , Aneta Siemiginowska $^{1}$, Matteo Guainazzi ${ }^{2}$, Martin Hardcastle $^{3}$, Giulia Migliori ${ }^{4}$ (1), \\ Luisa Ostorero $^{5}$ (D), and Łukasz Stawarz ${ }^{6}$ (D) \\ ${ }^{1}$ Center for Astrophysics, Harvard \& Smithsonian, 60 Garden Street, Cambridge, MA 02138, USA; msobolewska@cfa.harvard.edu \\ ${ }^{2}$ European Space Research and Technology Centre (ESA/ESTEC), Kepleriaan 1, 2201 AZ, Noordwijk, The Netherlands \\ ${ }^{3}$ School of Physics, Astronomy and Mathematics, University of Hertfordshire, College Lane, Hatfield AL10 9AB, UK \\ ${ }^{4}$ INAF, Istituto di Radio Astronomia di Bologna, Via P. Gobetti 101, I-40129 Bologna, Italy \\ ${ }^{5}$ Dipartimento di Fisica—Università degli Studi di Torino and Istituto Nazionale di Fisica Nucleare (INFN), Via P. Giuria 1, I-10125 Torino, Italy \\ ${ }^{6}$ Astronomical Observatory, Jagiellonian University, ul. Orla 171, 30-244 Kraków, Poland \\ Received 2018 April 10; revised 2018 October 24; accepted 2018 November 1; published 2019 January 23
}

\begin{abstract}
Compact symmetric objects (CSOs) show radio features such as jets, lobes, and hot spots that are contained within the central $1 \mathrm{kpc}$ region of their host galaxy. Thus, they are thought to be among the progenitors of large-scale radio galaxies. A debate regarding whether the CSOs are compact primarily because they are young or because they are surrounded by a dense medium impacting their expansion is ongoing. Until now, attempts to discriminate between the environmental and genuine youthfulness scenarios have been inconclusive. We present a study of three CSOs selected on the basis of their puzzling X-ray absorbing properties in prior Beppo-SAX and/or Chandra $X$-ray Observatory data. Our new XMM-Newton observations unambiguously confirm the nature of their X-ray absorbers. Furthermore, for the first time, our X-ray data reveal the existence of a population of CSOs with intrinsic hydrogen column density $N_{\mathrm{H}}>10^{23} \mathrm{~cm}^{-2}$ that is different from the population of X-ray unabsorbed CSOs. The two groups appear to be separated in the linear size versus radio power plane. This finding suggests that a dense medium in X-ray obscured CSOs may be able to confine the radio jets. Alternatively, X-ray obscured CSOs could be seen as radio brighter than their unobscured counterparts either because they reside in a dense environment or because they have larger jet powers. Our results help constrain the origin of the X-ray emission and the location and size of the X-ray obscurer in CSOs, and indicate that the environment may play a key role during the initial expansion of a radio source.
\end{abstract}

Key words: galaxies: active - galaxies: evolution - galaxies: jets - X-rays: galaxies

\section{Introduction}

Gigahertz peaked-spectrum (GPS) radio sources are characterized by a spectral turnover at gigahertz frequencies, and compact sub-kiloparsec-scale radio structures (e.g., review in O'Dea 1998). A subclass of compact symmetric objects (CSOs) is identified by symmetric double radio structures with the radio intensity dominated by lobes or hot spots. These features are also typically observed in large-scale radio galaxies, but in the CSOs they are contained within the central $1 \mathrm{kpc}$ region of the host galaxy. Because by definition CSOs are expected to be observed in the direction nearly perpendicular to the radio jet axis, the observed emission is not affected by beaming. Thus, their linear radio size can be translated into the source kinematic age if one measures the advance velocity of the hot spots. Multiple studies show that the CSO radio structures may be very young, <3000 years old (e.g., Gugliucci et al. 2005; see also An \& Baan 2012, and references therein).

The reasons for the CSO compactness are still debated. Fanti et al. (1990) observed an anticorrelation between the radio turnover frequency and linear radio size in radio galaxies with linear sizes $<20 \mathrm{kpc}$. This finding, along with CSO radio morphologies resembling miniature versions of those seen in the FRI/FRII galaxies (Fanaroff \& Riley 1974) led to the formulation of an evolutionary scenario in which CSOs are the young counterparts of large-scale radio sources (e.g., O'Dea 1998; Snellen et al. 2000; Kunert-Bajraszewska et al. 2010). However, there is also increasing observational evidence in favor of the confinement scenario, in which the CSOs cannot expand freely because they reside in a dense environment (e.g., van Breugel et al. 1984; Bicknell et al. 1997; Callingham et al. 2015; Tingay et al. 2015). In particular, recent radio monitoring of CSOs provide observational evidence in favor of the existence of clouds with a complex density distribution in some sources (Callingham et al. 2015, 2017; Tingay et al. 2015).

X-ray observations have the potential to resolve this puzzle because the host galaxy environment can be studied in the $\mathrm{X}$-ray band via direct measurements of both the interstellar medium (ISM) temperature and the total equivalent hydrogen column density, $N_{\mathrm{H}}$, along the line of sight (see Siemiginowska et al. 2008; Siemiginowska 2009; Tengstrand et al. 2009 for reviews on X-ray emission of GPS sources). In Siemiginowska et al. (2016; hereafter S16) we presented the first X-ray study of a sample that included all CSOs with redshift $z<1$ and measured kinematic ages known by 2010 (16 sources; 6 of them we observed with Chandra $^{7}$ for the first time in X-rays). The majority of the observations were short, but indicated a diverse environment and moderate column densities. Radio surveys continue providing new CSO kinematic age estimates, and new CSOs and CSO candidate identifications (e.g., An \& Baan 2012; Tremblay et al. 2016).

S16 found that the majority of CSOs observed in X-rays show moderate or little intrinsic X-ray absorption (i.e., hydrogen equivalent column density of $N_{\mathrm{H}}<10^{22} \mathrm{~cm}^{-2}$ ). However, observations of a few sources suggested that they have a complex X-ray spectrum with a heterogeneous absorbing medium and required high quality data for detailed

Chandra X-ray Observatory. 
Table 1

Log of XMM-Newton Observations

\begin{tabular}{|c|c|c|c|c|c|}
\hline$\#$ & Source & R.A. & Decl. & Date & $\begin{array}{c}\text { Exposure }^{\mathrm{a}} \\
\text { PN/MOS1/MOS2 } \\
(\mathrm{ks})\end{array}$ \\
\hline 1 & $2021+614$ & 202206.682 & +613658.79 & 2016 May 25 & $24.5 / 30.6 / 36.0$ \\
\hline 2 & $1934-63$ & 193925.027 & -634245.61 & 2017 Apr 1 & $22.8 / 27.3 / 27.9$ \\
\hline 3 & $1946+708$ & 194553.520 & +705548.72 & 2016 Oct 21 & $18.1 / 24.9 / 26.3$ \\
\hline
\end{tabular}

Note.

${ }^{\text {a }}$ Effective exposure after applying the standard filtering criteria.

studies of their emission and absorption properties. In particular, the X-ray spectrum of OQ+208 observed with XMM-Newton (Guainazzi et al. 2004), and Chandra and NuSTAR (Sobolewska et al. 2018) is dominated by emission reflected from neutral matter. The direct power-law emission is intrinsically absorbed with a column density of $N_{\mathrm{H}}=$ (44-130) $\times 10^{23} \mathrm{~cm}^{-2}$ (Sobolewska et al. 2018). Thus, OQ +208 resembles an archetypal highly obscured, possibly Compton-thick AGN.

Vink et al. (2006) reported a significant amount of intrinsic $\mathrm{X}$-ray absorption in the XMM-Newton spectrum of another CSO, $0108+388\left(N_{\mathrm{H}}=5.7 \pm 2.0 \times 10^{23} \mathrm{~cm}^{-2}\right.$ for the photon index fixed at $\Gamma=1.75)$. Singh et al. (2011) found that $X M M$ Newton data of $0048+3157$ required three intrinsic absorbers with a range of hydrogen column densities; in particular, a partial coverer with $N_{\mathrm{H}} \sim 10^{23} \mathrm{~cm}^{-2}$. Risaliti et al. (2003) reported a Compton-thick absorption in two CSO sources studied with Beppo-SAX: 1934-63 and 1946+708. However, these two sources appeared Compton thin in the Chandra observations of S16. Similarly, $1607+26$ was classified as a Compton-thick source in an XMM-Newton observation with poor signal-to-noise ratio (S/N; Tengstrand et al. 2009), while S16 found $N_{\mathrm{H}}<1.8 \times 10^{21} \mathrm{~cm}^{-2}$ in a deeper $40 \mathrm{ks}$ Chandra observation. Finally, in S16 we reported a photon index of $\Gamma \sim 0.8-1.0$ in $1511+0518$ and $2021+614$, which is unusually hard for non-beamed sources. Both sources were flagged as Compton-thick CSO candidates.

We observed with XMM-Newton the two CSOs with prior Beppo-SAX and Chandra data (1934-63 and 1946+708) and one of the two Compton-thick candidates identified in S16 $(2021+614)$. Our goal was to constrain the presence of any line emission attributable to $\mathrm{Fe} \mathrm{K} \alpha$ originating from the locations of these three sources, constrain their X-ray absorption properties, and study any underlying correlations between the radio size, radio power, and CSO environment.

In this paper we present the results of our new XMM-Newton observations. We discuss the current status of X-ray absorption measurements in the S16 CSO sample, and the implications for the environment and initial expansion of radio sources.

\section{Observations}

Table 1 contains the details of our XMM-Newton observations. We present the data obtained with the EPIC instruments. We used the standard XMM-Newton pipeline products (PPS event lists processed with SAS v.15.0 for 2021+614 and 1946 +708 , and SAS v.16.0 for 1934-63). We applied the standard screening of the events, excluding the periods of flaring particle background. The resulted clean exposure times for each source are listed in Table 1 . We note that the high background
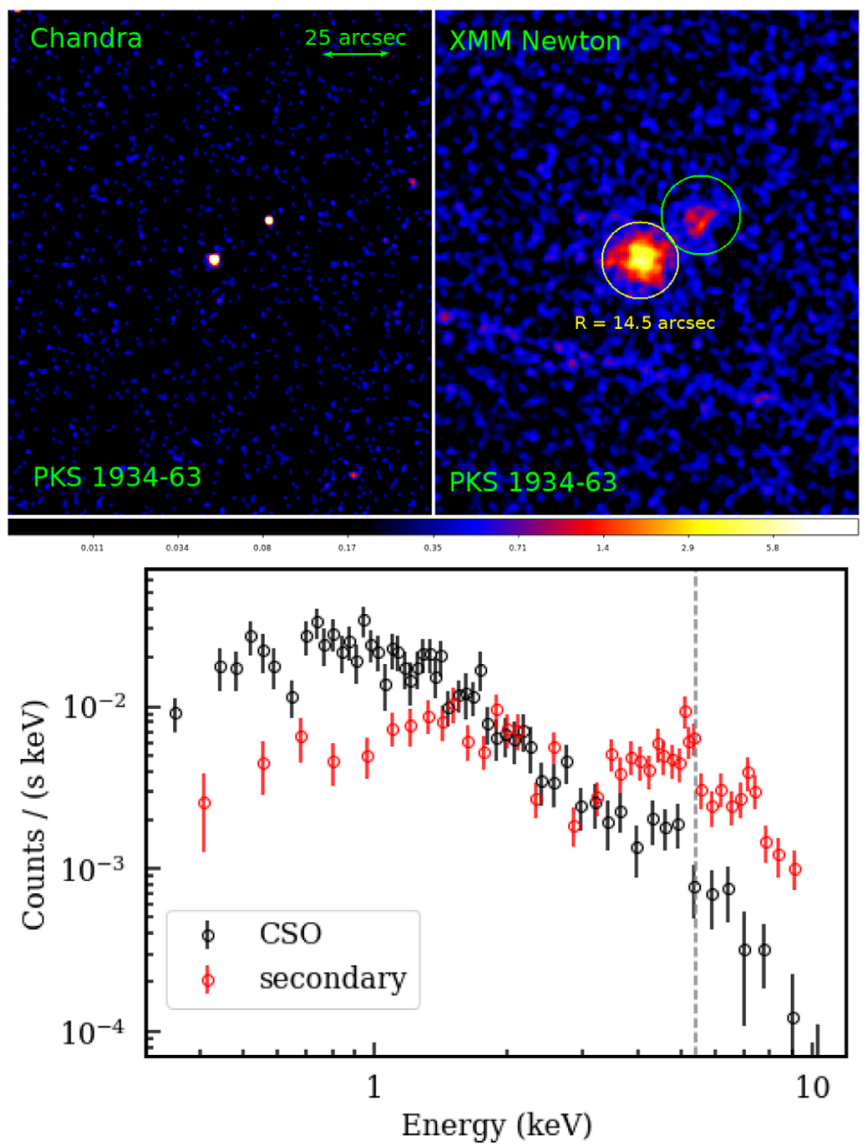

Figure 1. Top: Chandra ACIS-S (top/left) and XMM-Newton EPIC PN (top/ right) images centered on 1934-63. Yellow and green circles mark the XMMNewton extraction regions for 1934-63 and the serendipitous source, respectively. The Chandra image is smoothed with a Gaussian kernel with 1". 5 radius. The XMM-Newton image is binned by 16 pixels and smoothed with a Gaussian kernel with a radius of 2 ". 4 . The logarithmic color scale corresponds to the XMM-Newton image. Bottom: XMM-Newton PN data corresponding to $1934-63$ (black) and the secondary source (red). The vertical line marks the energy of the Fe $\mathrm{K} \alpha$ line redshifted to the frame of 1934-63.

rates impacted approximately half of the originally scheduled exposure of $54 \mathrm{ks}$ for $2021+614$.

Our previous $20 \mathrm{ks}$ Chandra observation revealed a background X-ray source 25" away from 1934-63 in the NW direction (Figure 1, top/left; S16). This source is also visible in our XMM-Newton image. Thus, we chose 14 ". 5 and $15^{\prime \prime}$ extraction regions for 1934-63 and the secondary source, respectively, to avoid mutual flux contamination. There are no contaminating sources in the other two CSOs and the standard circular regions with the radius of $24^{\prime \prime}$ were used for extracting spectra of $2021+614$ and $1946+708$. 
Table 2

Parameters of the Best-fitting Spectral Models

\begin{tabular}{|c|c|c|c|c|c|c|}
\hline $\begin{array}{l}\text { Parameter } \\
\text { (1) }\end{array}$ & $\begin{array}{l}\text { Unit } \\
\text { (2) }\end{array}$ & $\begin{array}{c}\text { 1934-63 } \\
(3) \\
\text { powerlaw }\end{array}$ & $\begin{array}{c}1946+708 \\
(4) \\
\text { powerlaw }\end{array}$ & \multicolumn{3}{|c|}{$2021+614$} \\
\hline$N_{\mathrm{H}}^{\mathrm{gaal}}$ & $10^{20} \mathrm{~cm}^{-2}$ & 6.16 & 8.57 & 14.01 & 14.01 & 14.01 \\
\hline$N_{\mathrm{H}}^{1}$ & $10^{21} \mathrm{~cm}^{-2}$ & $1.2_{-0.4}^{+0.8}$ & $16_{-3}^{+5}$ & $4_{-2}^{+2}$ & $4_{-1}^{+3}$ & $4.2_{-1.0}^{+0.6}$ \\
\hline$N_{\mathrm{H}}^{2}$ & $10^{23} \mathrm{~cm}^{-2}$ & $\ldots$ & $\ldots$ & $3.4_{-0.3}^{+0.3}$ & $3.5_{-0.8}^{+0.9}$ & $3.6_{-0.6}^{+0.5}$ \\
\hline$\Omega / 2 \pi$ & & $\ldots$ & $\ldots$ & 1(f) & $\ldots$ & $\ldots$ \\
\hline$C F_{\text {tor }}$ & & $\ldots$ & $\ldots$ & $\ldots$ & $0.5(f)$ & $0.6_{-0.4}^{+0.2}$ \\
\hline$C_{\mathrm{pl}}$ & & $\ldots$ & $\ldots$ & $0.12_{-0.06}^{+0.02}$ & $0.12_{-0.05}^{+0.03}$ & $0.08_{-0.03}^{+0.02}$ \\
\hline$E_{\mathrm{Fe}}$ & $\mathrm{keV}$ & $\ldots$ & $\ldots$ & $6.4(f)$ & $6.4(\mathrm{f})$ & $6.4(f)$ \\
\hline $\mathrm{EW}^{\mathrm{a}}$ & $\mathrm{keV}$ & $<0.3$ & $<0.2$ & $\ldots$ & $\sim 0.2$ & $\sim 0.2$ \\
\hline d.o.f. ${ }^{d}$ & & 269 & 51 & 69 & 68 & 68 \\
\hline
\end{tabular}

Notes. Errors represent $1 \sigma$ confidence intervals, except for EW and flux for which we give $90 \%$ confidence interval uncertainties. The cutoff energy was fixed at 100 , 500 , and $300 \mathrm{keV}$ in models presented in columns (5)-(7), respectively, but this has no impact on modeling the $<10 \mathrm{keV}$ XMM-Newton data.

${ }^{a}$ Equivalent width of the $\mathrm{Fe} \mathrm{K} \alpha$ emission line (PN) computed with respect to the total continuum.

${ }^{\mathrm{b}}$ Observed (not corrected for absorption) 2-10 keV (observed frame) flux (PN instrument).

${ }^{\mathrm{c}}$ Intrinsic (corrected for absorption) $2-10 \mathrm{keV}$ (rest-frame) luminosity of the direct power-law (PN instrument).

${ }^{\mathrm{d}}$ Degrees of freedom.

We fitted simultaneously the EPIC PN, MOS1, and MOS2 spectra using XSPEC v12.9.1s (Arnaud 1996).

\section{Results}

Our XMM-Newton observations enabled us to refine the intrinsic X-ray absorption measurements of our targets. We confirm that, in agreement with our prior Chandra observations (S16), the X-ray spectra of $1934-63$ and $1946+708$ can be described with a power-law model modified by absorption, $M_{\mathrm{abs}}^{1}=\exp \left(-N_{\mathrm{H}}^{\mathrm{gal}} \sigma_{E}\right) \exp \left(-N_{\mathrm{H}}^{1} \sigma_{E(1+z)}\right)$, where $\sigma_{E}$ is the photoelectric cross-section and $N_{\mathrm{H}}^{\mathrm{gal}}$ and $N_{\mathrm{H}}^{1}$ are the Galactic and intrinsic X-ray absorbing columns, respectively (phabs and zphabs XSPEC models). We detected only a mild intrinsic X-ray absorbing hydrogen column in both sources. By contrast, we show that $2021+614$, a source reported to be a Compton-thick candidate in S16, is indeed absorbed due to a significant intrinsic hydrogen column density. Parameters of our best-fitting X-ray spectral models are listed in Table 2 and the modeling details are given below.

\section{1. $1934-63$}

We detected an X-ray source adjacent to 1934-63 in both Chandra and XMM-Newton images (Figure 1, top). We were not able to model the Chandra X-ray spectrum of the secondary source due to a low $\mathrm{S} / \mathrm{N}$.

In Figure 1, bottom, we plot the EPIC PN spectra for the CSO and the secondary source. This comparison shows that the iron emission originates from the secondary source, while the XMM-Newton spectrum of the CSO is modeled well with an intrinsically absorbed power law (Table 2; see Figure 2 for the data to model ratio). Although the XMM-Newton data of the
CSO appear to contain a hint of iron emission at $\sim 6.5 \mathrm{keV}$ (rest frame), we only find an upper limit on the line's equivalent width, EW $<0.3 \mathrm{keV}$ ( $90 \%$ confidence level). We conclude that the two sources were blended in the Beppo-SAX observation reported by Risaliti et al. (2003) and this led to the incorrect suggestion that 1934-63 may contain a Comptonthick obscurer. Here we derived $N_{\mathrm{H}}^{1}=1.2_{-0.4}^{+0.8} \times 10^{21} \mathrm{~cm}^{-2}$ for 1934-63, consistent with our Chandra result (see S16).

\section{2. $1946+708$}

XMM-Newton data are consistent with our prior Chandra data (S16) and indicate that $1946+708$ is not a Compton-thick source. No nearby secondary X-ray sources are seen in our data. The source flux in our observations is comparable (within a factor of 2) with the flux reported by Risaliti et al. (2003) based on the Beppo-SAX data. Thus, we can now rule out a scenario considered in S16 in which the source switches between the Compton-thick and Compton-thin states on timescales of several years. We conclude that, most likely, the marginal detection of an iron line emission in the BeppoSAX data that prompted the Compton-thick interpretation was a false positive. In our XMM-Newton data the $90 \%$ confidence level on the equivalent width of the $6.4 \mathrm{keV}$ line at the redshift of the source is EW $<0.2 \mathrm{keV}$. With the much better quality $X M M-N e w t o n$ and Chandra data it is evident that the source is only mildly intrinsically absorbed $\left(N_{\mathrm{H}}^{1}=1.6_{-0.3}^{+0.5} \times 10^{22} \mathrm{~cm}^{-2}\right.$; Table 2; Figure 2).

\section{3. $2021+614$}

Application of an intrinsically absorbed power-law model to the XMM-Newton spectrum of $2021+614$ confirmed the 

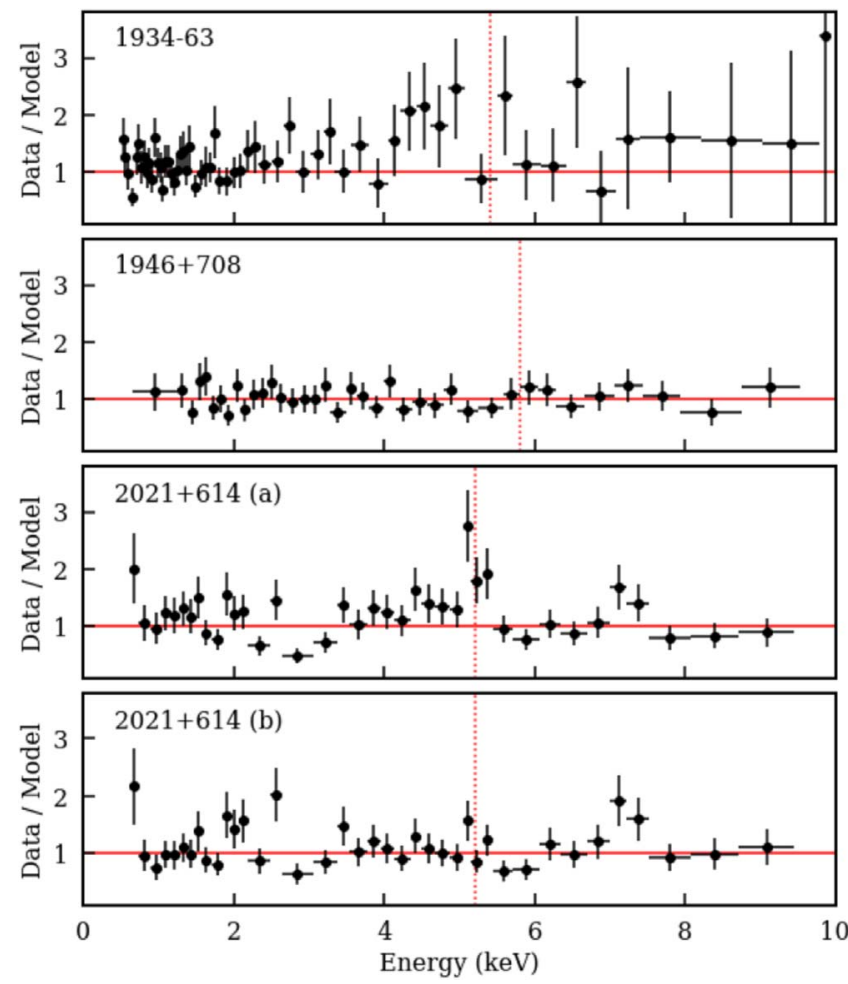

Figure 2. Ratios of the PN data to model. Top to bottom: 1934-63, power law modified with Galactic and intrinsic absorbing columns, Table 2, column (3); 1946 +708 , power law modified with Galactic and intrinsic absorbing columns, Table 2, column (4); 2021+614 (a), power law with Galactic and intrinsic absorbing columns, with no line emission; $2021+614$ (b), the torus model by Baloković et al. (2018), see Table 2, column (7); models presented for $2021+614$ in Table 2 columns (5) and (6) have data/model ratios that match closely those of $2021+614$ (b). Vertical lines mark the rest-frame energy of the FeK $\alpha$ emission detected only in $2021+614$

unusually hard photon index seen previously in our Chandra data (S16). In addition, we observed residuals near the region corresponding to the rest-frame $6.4 \mathrm{keV}$ energy, suggestive of the presence of the Fe K $\alpha$ fluorescent line emission (Figure 2; line emission was not required by our Chandra data, most likely due to low $\mathrm{S} / \mathrm{N}$ ratio at energies $>5 \mathrm{keV}$ ). When we added a Gaussian line to the model, we obtained the rest-frame energy of the line $E=6.3_{-0.8}^{+0.2} \mathrm{keV}$, equivalent width $\mathrm{EW}=$ $0.41_{-0.14}^{+0.08} \mathrm{keV}, \Gamma=0.34_{-0.06}^{+0.05}$, and $\chi^{2}=104$ for 69 degrees of freedom. The apparent hard photon index and the detection of the $\mathrm{Fe} \mathrm{K} \alpha$ line suggest strong intrinsic obscuration in this source.

To verify this, we constructed a model consisting of an intrinsically absorbed power law and its reflection from a neutral matter in the slab geometry (pexmon; Nandra et al. 2007) with the Fe $\mathrm{K} \alpha$ emission computed self-consistently. However, this model was not able to provide a good fit to the data for a range of typical AGN photon indices (e.g., Corral et al. 2011; Del Moro et al. 2017). Thus, we added a contribution from the direct power law that is scattered into the line of sight, rather than absorbed, by the obscuring material and dominates the spectrum at energies below $\sim 3 \mathrm{keV}$. The total model was given by $M_{\mathrm{abs}}^{1} \times\left[C_{\mathrm{pl}} \times\right.$ cutoffpl $+\exp \left(-N_{\mathrm{H}}^{2} \sigma_{E(1+z)}\right) \times$ pexmon $]$, with the second intrinsic absorber modifying only the pexmon component. Note that with our choice of the reflection amplitude, $\Omega / 2 \pi$, fixed at 1 , the pexmon component stands for the combination of the direct cutoffpl and reflected continuum, including the line emission. The photon indices and normalizations of the scattered cutoffpl and pexmon components were linked to each other, and the cutoff energy was fixed at $100 \mathrm{keV}$. We obtained a very good fit with $\chi^{2}=73$ for 67 degrees of freedom, $\Gamma=1.6 \pm 0.2$, and two intrinsic absorbing columns of the order of $10^{21} \mathrm{~cm}^{-2}$ and $10^{23} \mathrm{~cm}^{-2}$ (Figure 3, left; Table 2, column (5)). The ratio of the scattered and direct power-law normalizations was rather high, $C_{\mathrm{pl}}=$ $0.14_{-0.06}^{+0.07}$ (e.g., Ueda et al. 2007; Marchesi et al. 2018).

Finally, we explored two models of a toroidal reprocessor (mytorus, Yaqoob 2012; borus02, Baloković et al. 2018). The key difference between these two models is the flexibility of the borus 02 model to fit for the torus covering factor, $C F_{\text {tor }}$, fixed at 0.5 in the mytorus model. The total model was defined as $M_{\mathrm{abs}}^{1} \times\left[C_{\mathrm{pl}} \times\right.$ cutoffpl $+M_{\mathrm{abs}}^{2} \times$ cutoffpl $+A \times$ torus $]$, with $M_{\mathrm{abs}}^{2}=\exp \left[-N_{\mathrm{H}}^{2}(\theta) \sigma_{E(1+z)}\right]$ being the zeroth-order continuum given by Yaqoob (2012). We used the relevant reflected and line emission table models provided by Yaqoob (2012) and Baloković et al. (2018) to express the torus component. In both cases the line-of-sight column density was tied to the average toroidal column density. The inclination angle was fixed at $80^{\circ}$ since the CSO sources are believed to be viewed edge on, through the obscuring torus, given the symmetricity of their radio structures and underlying assumption that the jet axis is perpendicular to the plane of the torus. We introduced a normalization constant $A$ following Yaqoob (2012) who argue that the flexibility in modeling the relative normalizations of the scattered and line emission is required in order to account for effects such as a deviation from a perfect toroidal geometry, a range of covering factors, etc. It was a fit parameter in the mytorus model $(A=2.3 \pm 1.2)$ and it was fixed at 1 (by construction) in the borus 02 model. We found good fits for both toroidal models, and the resulting model parameters were consistent between the two approaches (Figure 3, middle/right; Table 2, columns (6-7)). Fit parameters of the toroidal models were consistent with those of the pexmon model. The toroidal models result in the deabsorbed $2-10 \mathrm{keV}$ rest-frame luminosity of the order of $10^{44} \mathrm{erg} \mathrm{s}^{-1}$, while the slab geometry model gives $\sim 6.5 \times 10^{43} \mathrm{erg} \mathrm{s}^{-1}$.

Models presented in Table 2, columns (5-7) are statistically equivalent given the current data, and result in the ratio of the data to model of comparable quality and energy dependence (illustrated in the bottom panel in Figure 2). However, all these models imply that the nuclear X-ray emission of $2021+614$ is seen through a matter with high intrinsic X-ray absorbing column, $N_{\mathrm{H}}^{2} \simeq 3.5 \times 10^{23} \mathrm{~cm}^{-2}$, and another intrinsic absorber with $N_{\mathrm{H}}^{1}$ of the order of $10^{21} \mathrm{~cm}^{-2}$.

\subsection{Subpopulation of X-Ray Absorbed CSOs}

In Table 3 and Figure 4 we combined information on the intrinsic X-ray absorption in CSOs, CSO radio power at $5 \mathrm{GHz}$, and radio source linear size. We stress that sources in Table 3 represent all currently known CSOs observed both in the radio and X-ray bands, and $N_{\mathrm{H}}(z)$ listed in column 7 indicates the highest intrinsic equivalent column density detected in the $\mathrm{X}$-ray band in each source. We used the radio data provided by Tremblay et al. (2016), and for sources from An \& Baan (2012) we derived the $5 \mathrm{GHz}$ radio power from their spectral index, $\alpha$, and $4.8 \mathrm{GHz}$ flux as $L_{5 \mathrm{GHz}} \simeq 4 \pi S_{4.8} D_{L}^{2} /(1+z)^{1-\alpha}$, where $D_{L}$ is the luminosity distance to the source assuming WMAP9 cosmology (Hinshaw et al. 2013). The X-ray 

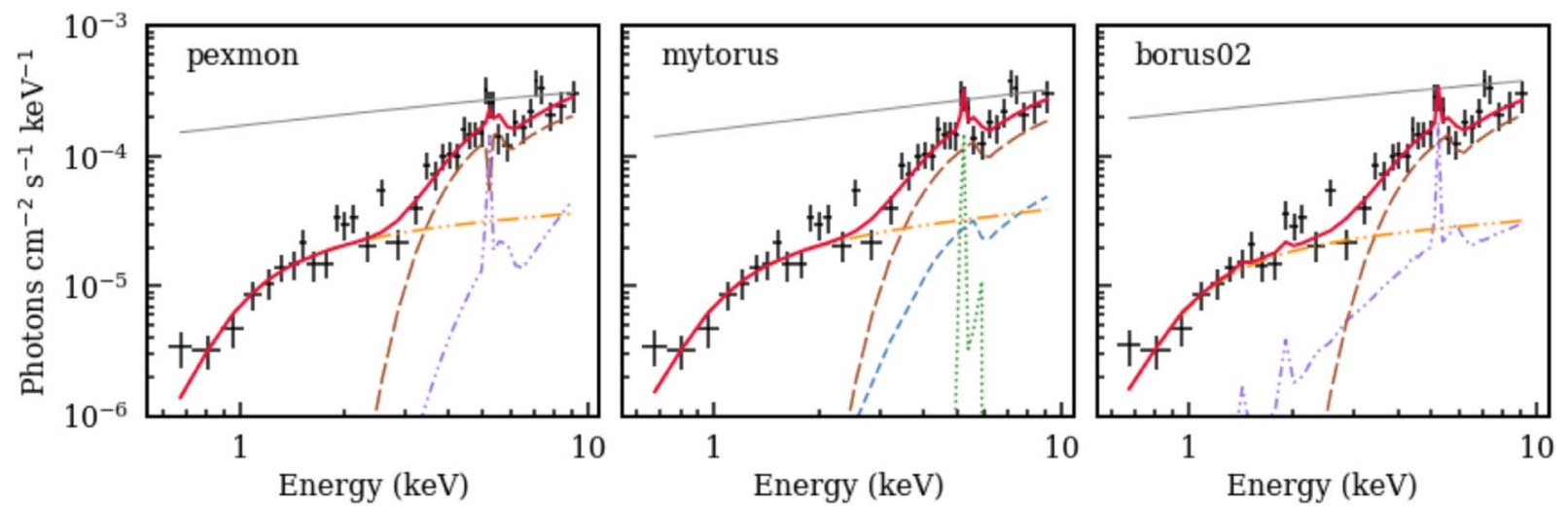

Figure 3. Best-fit "unfolded" models (red/solid line) and the PN data of 2021+614 (crosses), and model components. Left: reflection from a slab, Table 2, column (5). Middle/Right: reflection from a torus, Table 2, columns (6)/(7). The model components are as follows: intrinsically absorbed direct continuum (brown/long-dashed), and scattered direct continuum (orange/dotted-long-dashed). Reflection from a torus and the fluorescent FeK $\alpha$ line emission are computed and plotted jointly in the case of the pexmon and borus 02 models (purple/dotted-short-dashed), and separately in the case of the mytorus model (blue/short-dashed and green/dotted, respectively). Thin solid gray lines show the deabsorbed direct power-law emission.

exposures are relatively deep, with 8 sources observed for $>30 \mathrm{ks}, 11$ sources observed for 9-30 ks, and only 5 sources observed for $2-5 \mathrm{ks}$.

We found that intrinsically X-ray unabsorbed and X-ray absorbed CSOs $\left(N_{\mathrm{H}}<10^{23} \mathrm{~cm}^{-2}\right.$ and $N_{\mathrm{H}}>10^{23} \mathrm{~cm}^{-2}$, respectively) appear to be separated in the size of the radio source versus $5 \mathrm{GHz}$ luminosity plane (Figure 4). In general, the former cluster around the relation reported by Tremblay et al. (2016 see also Orienti \& Dallacasa 2014), while the latter are located below this correlation. To estimate the statistical significance of this separation we performed two one-dimensional two-sample Kolmogorov-Smirnov (K-S) tests with respect to the CSO radio luminosity and radio size. The results suggest that while we cannot reject the hypothesis that the radio luminosities of the two samples come from the same distribution $(\mathrm{KS}=0.3$ and $p$-value $=0.8$ ), the radio sizes of the two samples mostly do not come from the same distributions at a significance level $>99 \%$ $\left(\mathrm{KS}=0.75\right.$ and $p$-value $\left.=9.8 \times 10^{-4}\right)$. This would support our conclusion that the obscured and unobscured sources appear to have different radio sizes.

On the other hand, a two-dimensional two-sample K-S test should be used in the statistical verification of our results (e.g., Peacock 1983; Fasano \& Franceschini 1987). However, this test requires that the size of each sample is larger than $\sim 10$. This condition is not met by our current data as we have only five obscured CSOs. The two-dimensional two-sample K-S test can be successfully performed in the future, when X-ray data become available for an extended CSO sample. Nevertheless, for our current unabsorbed and absorbed samples the twodimensional two-sample $\mathrm{K}-\mathrm{S}$ test results in $\mathrm{KS}=0.8$ and $p$-value $=0.02$, which, if confirmed with future data, would suggest that at a $98 \%$ confidence level the low- and high- $N_{\mathrm{H}}$ CSOs occupy different regions in the radio size versus radio luminosity plane.

We conclude that our data are suggestive of the first observational evidence in the X-ray band for a statistically significant separation in the CSO population with respect to the density of the CSO environment, as measured through the intrinsic equivalent hydrogen column density, and radio properties such as the source size, and perhaps also source radio luminosity.

\section{Discussion}

We observed three Compton-thick CSO candidates with $X M M-N e w t o n$ and were able to refute the presence of a Compton-thick absorber in 1934-63 and 1946+708, and detect a significant intrinsic X-ray absorbing column in 2021 +614 . The X-ray spectrum of $2021+614$ shows that the intrinsic X-ray power law is strongly attenuated and it is seen primarily in a reflection from a neutral matter. In particular, the detection of the $6.4 \mathrm{keV}$ iron emission line accompanying the reflection continuum indicates that $2021+614$ is not a beamed source (see Giommi et al. 2007 and D'Abrusco et al. 2014 who classified the source as a blazar). Our findings are in agreement with the results of Bartel et al. (1984) who reported on strong reddening in the optical spectroscopic observations of 2021 +614 performed with the Multiple Mirror Telescope.

We note that the best-fit photon indexes of the power-law models applied to the X-ray spectra of these three CSOs cover a range of $\Gamma \sim 1-2$ (Table 2). Thus, it is likely that different physical processes give rise to the intrinsic X-ray emission in these sources (e.g., inverse-Compton processes in expanding radio lobes, X-ray jet, X-ray corona).

We observed that the X-ray absorbed and unabsorbed CSOs appear to be separated in the radio size versus $5 \mathrm{GHz}$ radio luminosity plane (Figure 4). We performed one-dimensional two-sample K-S tests with respect to the radio properties, and found that the radio sizes of the absorbed and unabsorbed CSO samples could form two populations. In addition, the results of a two-dimensional two-sample K-S test confirmed the possible existence of such two populations. However, we caution that the two-dimensional two-sample K-S test requires a larger number of the absorbed sources (at least twice as many as in the current sample) to be valid.

Our results could be interpreted in two ways. It could be that the CSOs with a high intrinsic X-ray absorbing column, $N_{\mathrm{H}}>$ $10^{23} \mathrm{~cm}^{-2} ; 0048+3157, \mathrm{OQ}+208,2021+614,1511+518$, $0108+388$ ) have less evolved radio structures (smaller linear radio size) than X-ray unabsorbed CSOs with the same $5 \mathrm{GHz}$ radio power. Thus, the environment in which a radio source expands might be able to confine the source growth already at the earliest stages of its evolution. This would provide the first observational evidence in the $\mathrm{X}$-ray band that some compact 
Table 3

X-Ray Observed CSO Sources

\begin{tabular}{|c|c|c|c|c|c|c|c|c|c|}
\hline (1) & $\begin{array}{c}\text { Source } \\
\text { (2) }\end{array}$ & (3) & $\begin{array}{l}\text { Size } \\
(\mathrm{pc}) \\
(4)\end{array}$ & $\begin{array}{c}\log L(5 \mathrm{GHz}) \\
\left(\mathrm{W} \mathrm{m}^{-2}\right) \\
(5)\end{array}$ & $\begin{array}{l}\text { References } \\
\text { (radio) } \\
\text { (6) }\end{array}$ & $\begin{array}{c}N_{\mathrm{H}}(z) \\
10^{22} \mathrm{~cm}^{-2} \\
(7)\end{array}$ & $\begin{array}{l}\Gamma \\
(8)\end{array}$ & $\begin{array}{l}\text { X-ray } \\
\text { Observatory } \\
\text { (9) }\end{array}$ & $\begin{array}{l}\text { References } \\
\text { (X-ray) } \\
\text { (10) }\end{array}$ \\
\hline 1 & $0035+227$ & 0.096 & 31.3 & 24.75 & [1] & $1.4_{-0.6}^{+0.8}$ & $(1.7)$ & Chandra & [3] \\
\hline 3 & $0710+439$ & 0.518 & 146 & 27.16 & [1] & $1.02_{-0.22}^{+0.29}$ & $1.75_{-0.10}^{+0.11}$ & Chandra & [3] \\
\hline 4 & $1031+567$ & 0.460 & 181.8 & 26.98 & [1] & $0.50 \pm 0.18$ & $(1.75)$ & XMM-Newton & [4] \\
\hline 5 & OQ+208 & 0.077 & 11.1 & 25.55 & [1] & 44-130 & $1.448_{-0.002}^{+0.128}$ & XMM-Newton/Chandra/NuSTAR & [5] \\
\hline 8 & $1718-649$ & 0.014 & 2.8 & 24.34 & [1] & $0.31-0.69$ & $1.75_{-0.09}^{+0.10}$ & Chandra/XMM-Newton & {$[3,6]$} \\
\hline 9 & $1843+356$ & 0.764 & 31 & 27.25 & [1] & $0.8_{-0.7}^{+0.9}$ & (1.7) & Chandra & {$[3]$} \\
\hline 10 & $1934-63^{c}$ & 0.181 & 128 & 26.79 & [1] & $0.12_{-0.04}^{+0.08}$ & $2.07_{-0.07}^{+0.20}$ & Chandra/XMM-Newton & This work \\
\hline 11 & $1943+546$ & 0.263 & 165 & 26.29 & [1] & $1.1 \pm 0.7$ & (1.7) & Chandra & {$[3]$} \\
\hline 12 & $1946+708^{c}$ & 0.101 & 57 & 25.21 & [1] & $1.6_{-0.3}^{+0.5}$ & $1.1_{-0.1}^{+0.3}$ & Chandra/XMM-Newton & This work \\
\hline 17 & $0431+2037$ & 0.219 & 145.6 & 26.47 & [1] & $<0.69$ & $0.63-2.62$ & $X M M-N e w t o n$ & [9] \\
\hline \multirow[t]{2}{*}{18} & $0503+0203(1)$ & 0.584 & 66.5 & 27.45 & [1] & $1.0_{-0.9}^{+1.1}$ & $2.0_{-0.5}^{+0.7}$ & $X M M-N e w t o n$ & [7] \\
\hline & $0503+0203(2)$ & 0.584 & 66.5 & 27.37 & [1] & $0.5_{-0.2}^{+0.3}$ & $1.62_{-0.19}^{+0.21}$ & XMM-Newton & [7] \\
\hline 19 & $1148+5254$ & 1.632 & 4.3 & 21.88 & [1] & $<0.73$ & $1.58 \pm 0.12$ & XMM-Newton & [10] \\
\hline 20 & $\mathrm{~J} 12201+2916$ & 0.002 & 1.5 & 21.88 & [2] & $<0.0678$ & $2.02-2.38$ & Chandra/XMM-Newton & [11] \\
\hline 21 & $\mathrm{~J} 12342+4753$ & 0.372 & 80.8 & 26.09 & [2] & $0.06_{-0.06}^{+0.12}$ & $1.80_{-0.20}^{+0.24}$ & Chandra & {$[12]$} \\
\hline 22 & $1256+5652$ & 0.042 & 56.0 & 24.24 & [1] & $8.35_{-4.64}^{+4.02}$ & $1.57_{-0.30}^{+0.14}$ & $X M M$-Newton & {$[8]$} \\
\hline 23 & $\mathrm{~J} 13262+3154$ & 0.368 & 285.4 & 26.95 & [2] & $0.12_{-0.05}^{+0.06}$ & $1.74 \pm 0.20$ & XMM-Newton & [9] \\
\hline 24 & $1400+6210$ & 0.431 & 218 & 26.80 & [9] & $2.9_{-1.0}^{+2.0}$ & $1.24 \pm 0.17$ & XMM-Newton & [9] \\
\hline
\end{tabular}

Notes.

a Compton-thick candidate (S16).

b Reported to be Compton thick in XMM-Newton data (Tengstrand et al. 2009). Much better quality Chandra data revealed that the source is unabsorbed.

${ }^{\mathrm{c}}$ Reported to be CT in Beppo-SAX data (Risaliti et al. 2003). Much better quality Chandra data revealed that the source is unabsorbed.

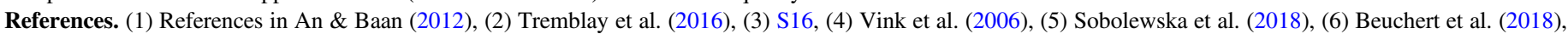
(7) Guainazzi et al. (2006), (8) Singh et al. (2011), (9) Tengstrand et al. (2009), (10) Young et al. (2009), (11) Younes et al. (2010), (12) Green et al. (2009).

jets may be frustrated by a dense environment and their kinematic ages may be underestimated.

An alternative way of explaining the separation visible in Figure 4 is that the X-ray obscured sources have higher radio luminosity than the $\mathrm{X}$-ray unobscured sources for a given radio size. The two factors that drive radio luminosity for a given size of a radio source are environmental density and jet power. Thus, one possible explanation is that the X-ray obscured sources are brighter for a given size because they were born in a denser environment. Another option is that they are brighter than the unobscured CSOs with the same size because they have higher jet power, which is related to central density, through e.g., the accretion rate (e.g., Allen et al. 2006; Tchekhovskoy et al. 2011; Ghisellini et al. 2014), or directly as in the model of Stawarz et al. (2008).

Even though with the current data it is not possible to distinguish between these two explanations, we stress that in both cases the density of the CSO environment plays a key role. Moreover, our results raise a question about the origins of the X-ray emission and the location of the X-ray obscurer. If the CSO X-ray emission originates primarily from an active nucleus that becomes attenuated by obscuring material in the vicinity of the nucleus, then we should also see heavily obscured CSOs with larger radio structures. In contrast, the CSOs in our sample show low intrinsic X-ray absorption for the radio size exceeding a few tens of parsecs. If the CSO X-ray emission is dominated by inverse-Compton emission of the radio lobes (e.g., Stawarz et al. 2008; Ostorero et al. 2010), then the CSOs would become unobscured once their radio lobes expand beyond the region occupied by the dense material. Thus, it could be that the obscurer is located on scales smaller than a few tens of parsecs. Alternatively, the obscuring material could be destroyed or removed by the expanding radio source.

Recently, Ostorero et al. (2017) addressed the question of the relative location of the X-ray and radio absorbers in the CSOs. They confirmed a positive correlation between the total absorbing column density $\left(N_{\mathrm{H}}, \mathrm{X}\right.$-rays) and the neutral hydrogen column $\left(N_{\mathrm{HI}}\right.$, radio), originally found by Ostorero et al. (2010). The existence of such a correlation suggests that the gas responsible for the X-ray and radio absorption may be part of the same, possibly unsettled, hundred-parsec-scale structure.

We note that there are three sources whose X-ray properties place them in the vicinity of the X-ray absorbed sources in Figure 4 and/or significantly below the correlation reported by Tremblay et al. (2016). Yet, they appear to be X-ray unabsorbed with $N_{\mathrm{H}}$ of the order of $10^{20}-10^{22} \mathrm{~cm}^{-2}$. We observed one of them with Chandra $\left(1843+356 ; L_{5 \mathrm{GHz}}=1.8 \times 10^{27} \mathrm{~W} \mathrm{~Hz}^{-1}, \mathrm{LS}=31 \mathrm{pc}\right)$ for $5 \mathrm{ks}$ and detected only $10.8 \pm 3.3$ counts. Thus, the measured 


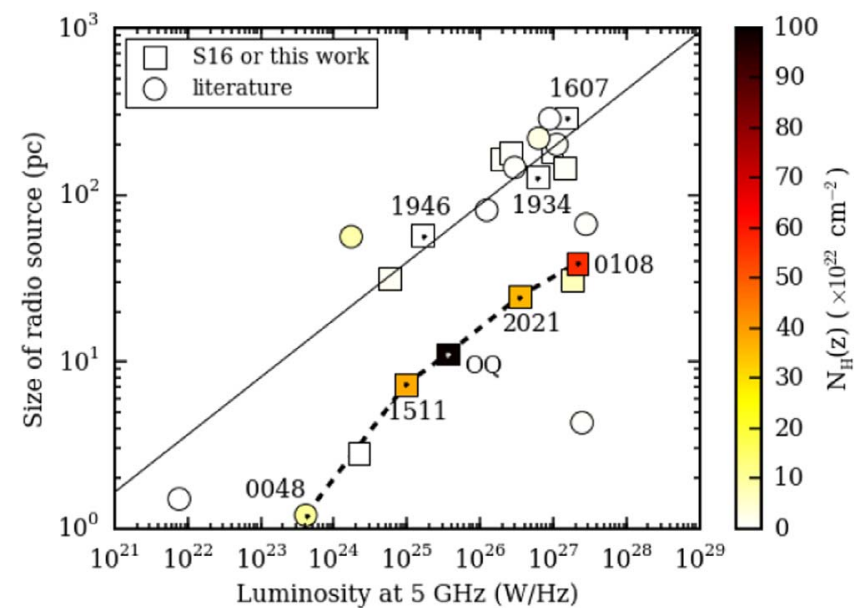

Figure 4. Luminosity at $5 \mathrm{GHz}$ vs. radio source size for CSOs observed with Chandra and/or XMM-Newton. Squares mark sources with kinematic age measurements studied in X-rays in S16 and this work. Circles mark new CSO or candidates (An \& Baan 2012; Tremblay et al. 2016) with X-ray information collected from the literature (Guainazzi et al. 2006; Vink et al. 2006; Green et al. 2009; Tengstrand et al. 2009; Young et al. 2009; Younes et al. 2010; Singh et al. 2011). Color coding represents the intrinsic equivalent hydrogen column density measured from the X-ray spectra. Solid line marks the relation fitted to the CSO data of Tremblay et al. (2016). Dashed line connects CSOs with intrinsic $N_{\mathrm{H}}>10^{23} \mathrm{~cm}^{-2}$ (labeled). Sources labeled as 1946, 1934, and 1607 have been previously reported to be Compton thick but verified to be only mildly absorbed in our XMM-Newton and/or Chandra observations (see the text for details).

intrinsic absorption is highly uncertain (S16). However, we should be able to robustly constrain both the $\Gamma$ and intrinsic $N_{\mathrm{H}}$ in this source with the pending XMM-Newton observation. The two remaining sources display characteristics that are not typical to our CSO X-ray sample. PKS $1718-649\left(L_{5 \mathrm{GHz}}=\right.$ $\left.2.2 \times 10^{24} \mathrm{~W} \mathrm{~Hz}^{-1}, \mathrm{LS}=2 \mathrm{pc}\right)$ is to date the only $\gamma$-ray emitting CSO (Migliori et al. 2016). While at $z=1.632,1148$ +5254 (a.k.a. SDSS J114856.56 $+525425.2 ; L_{5 \mathrm{GHz}}=2.4 \times$ $10^{27} \mathrm{~W} \mathrm{~Hz}^{-1}, \mathrm{LS}=4.3 \mathrm{pc}$ ) is the only source in Figure 4 with redshift above 1 . Additionally, its radio data indicate a presence of a larger scale (older) emission not associated with the younger radio source (Young et al. 2009; Tremblay et al. 2016).

The CSOs constitute the youngest resolved radio structures and provide information on early stages of the radio source expansion, and its impact on the feedback between the evolution of a galactic environment and black hole growth. The future X-ray observations of a large number of the CSOs are necessary for a statistical verification of the trends observed in this work.

\section{Conclusions}

We studied the X-ray and radio properties of a sample of CSOs. The sample included all currently identified CSOs with (1) X-ray spectral information obtained with Chandra and/or $X M M-N e w t o n,(2)$ morphological information on their compact radio structures, and (3) radio band spectral information. We considered a three-dimensional parameter space with axes corresponding to the radio luminosity at $5 \mathrm{GHz}, L_{5 \mathrm{GHz}}$, radio source linear size, LS, and intrinsic X-ray hydrogen column density, $N_{\mathrm{H}}$. Our main findings are as follows.

1. Two CSOs, 1934-63 and 1946+708, observed with XMMNewton are only mildly X-ray obscured indicating that they are not Compton-thick sources (dismissing the original claim based on the lower-quality BeppoSAX data), while the third CSO, 2021+614, is highly absorbed with the X-ray intrinsic column density of $\sim 3.5 \times 10^{23} \mathrm{~cm}^{-2}$.

2. X-ray obscured and unobscured CSOs appear to occupy separate regions in the $\left(L_{5 \mathrm{GHz}}, \mathrm{LS}, N_{\mathrm{H}}\right)$ parameter space.

3. One interpretation of the above separation is that X-ray absorbed CSOs have smaller radio sizes than X-ray unabsorbed CSOs. Alternatively, X-ray absorbed CSOs could be more radio luminous than X-ray unabsorbed CSOs with the same radio size.

4. We argued that in both cases the high density environment plays the key role: (1) High density ISM could prevent the radio source from expanding freely. (2) Radio source born in a dense environment would appear more radio luminous than a source born in a low density environment, as a high density environment is able to sustain a higher mass accretion rate onto a black hole than a low density environment. Consequently, jet power and hence radio luminosity increase with increasing mass accretion rate.

5. If X-ray emission originates in radio lobes, then our results place an upper limit on the distance of the high column density clouds from the center as we did not detect high intrinsic X-ray obscuration in CSOs with LS exceeding a few tens of parsecs.

Expanding our sample of X-ray CSOs is necessary in order to verify the statistical significance of the separation between the $\mathrm{X}$-ray obscured and unobscured CSO in the radio power versus radio size plane and draw important conclusions on the effect of the environment on the early evolution of the radio sources. Deep XMM-Newton exposures of $1511+518$ (Compton-thick candidate identified in S16) and $1843+356$ would allow us to put more stringent constraints on the intrinsic X-ray absorbers in these sources. Excellent candidates for a follow-up X-ray study, with radio properties that place them near the emerging X-ray absorbed branch in Figure 4, have recently been identified by An \& Baan (2012) and Tremblay et al. (2016). Ongoing and future radio surveys will keep providing new CSO targets for X-ray follow-ups.

The authors thank G. Risaliti and M. Baloković for discussions regarding spectral modeling of the studied sources. M.S. and A.S. were supported by NASA contract NAS8-03060 (Chandra X-ray Center). M.S. acknowledges the Polish NCN grant OPUS 2014/13/B/ST9/00570. Ł.S. was supported by the Polish NCN grant 2016/22/E/ST9/00061. This project was funded in part by the Chandra grant GO4-15099X. L.O. acknowledges partial support from the INFN Grant InDark and the grant of the Italian Ministry of Education, University and Research (MIUR) (L. 232/2016) "ECCELLENZA1822 D206-Dipartimento di Eccellenza 2018-2022 Fisica" awarded to the Dept. of Physics of the University of Torino. The XMM-Newton observations were obtained as part of the 078461 project.

\section{ORCID iDs}

Małgosia Sobolewska (ib https://orcid.org/0000-00026286-0159

Giulia Migliori (1D https://orcid.org/0000-0003-0216-8053

Luisa Ostorero (i) https://orcid.org/0000-0003-3983-5980

Łukasz Stawarz (i) https://orcid.org/0000-0001-8294-9479 


\section{References}

Allen, S. W., Dunn, R. J. H., Fabian, A. C., Taylor, G. B., \& Reynolds, C. S. 2006, MNRAS, 372, 21

An, T., \& Baan, W. A. 2012, ApJ, 760, 77

Arnaud, K. A. 1996, adass V, 101, 17

Baloković, M., Brightman, M., Harrison, F. A., et al. 2018, ApJ, 854, 42

Bartel, N., Shapiro, I. I., Huchra, J. P., \& Kuhr, H. 1984, ApJ, 279, 112

Beuchert, T., Rodríguez-Ardila, A., Moss, V. A., et al. 2018, A\&A, 612, L4

Bicknell, G. V., Dopita, M. A., \& O’Dea, C. P. O. 1997, ApJ, 485, 112

Callingham, J. R., Ekers, R. D., Gaensler, B. M., et al. 2017, ApJ, 836, 174

Callingham, J. R., Gaensler, B. M., Ekers, R. D., et al. 2015, ApJ, 809, 168

Corral, A., Della Ceca, R., Caccianiga, A., et al. 2011, A\&A, 530, A42

D’Abrusco, R., Massaro, F., Paggi, A., et al. 2014, ApJS, 215, 14

Del Moro, A., Alexander, D. M., Aird, J. A., et al. 2017, ApJ, 849, 57

Fanaroff, B. L., \& Riley, J. M. 1974, MNRAS, 167, 31P

Fanti, R., Fanti, C., Schilizzi, R. T., et al. 1990, A\&A, 231, 333

Fasano, G., \& Franceschini, A. 1987, MNRAS, 225, 155

Ghisellini, G., Tavecchio, F., Maraschi, L., Celotti, A., \& Sbarrato, T. 2014, Natur, 515, 376

Giommi, P., Capalbi, M., Cavazzuti, E., et al. 2007, A\&A, 468, 571

Green, P. J., Aldcroft, T. L., Richards, G. T., et al. 2009, ApJ, 690, 644

Guainazzi, M., Siemiginowska, A., Rodriguez-Pascual, P., \& Stanghellini, C. 2004, A\&A, 421, 461

Guainazzi, M., Siemiginowska, A., Stanghellini, C., et al. 2006, A\&A, 446, 87 Gugliucci, N. E., Taylor, G. B., Peck, A. B., \& Giroletti, M. 2005, ApJ, 622, 136 Hinshaw, G., Larson, D., Komatsu, E., et al. 2013, ApJS, 208, 19

Kunert-Bajraszewska, M., Gawroński, M. P., Labiano, A., \& Siemiginowska, A. 2010, MNRAS, 408, 2261
Marchesi, S., Ajello, M., Marcotulli, L., et al. 2018, ApJ, 854, 49

Migliori, G., Siemiginowska, A., Sobolewska, M., et al. 2016, ApJL, 821, L31

Nandra, K., O’Neill, P. M., George, I. M., \& Reeves, J. N. 2007, MNRAS, 382,194

O’Dea, C. P. 1998, PASP, 110, 493

Orienti, M., \& Dallacasa, D. 2014, MNRAS, 438, 463

Ostorero, L., Moderski, R., Stawarz, Ł., et al. 2010, ApJ, 715, 1071

Ostorero, L., Morganti, R., Diaferio, A., et al. 2017, ApJ, 849, 34

Peacock, J. A. 1983, MNRAS, 202, 615

Risaliti, G., Woltjer, L., \& Salvati, M. 2003, A\&A, 401, 895

Siemiginowska, A. 2009, AN, 330, 264

Siemiginowska, A., LaMassa, S., Aldcroft, T. L., Bechtold, J., \& Elvis, M. 2008, ApJ, 684, 811

Siemiginowska, A., Sobolewska, M., Migliori, G., et al. 2016, ApJ, 823, 57, S16

Singh, V., Shastri, P., \& Risaliti, G. 2011, A\&A, 532, A84

Snellen, I. A. G., Schilizzi, R. T., Miley, G. K., et al. 2000, MNRAS, 319, 445

Sobolewska, M., Siemiginowska, A., Guainazzi, M., et al. 2018, ApJ, submitted

Stawarz, Ł., Ostorero, L., Begelman, M. C., et al. 2008, ApJ, 680, 911

Tchekhovskoy, A., Narayan, R., \& McKinney, J. C. 2011, MNRAS, 418, L79

Tengstrand, O., Guainazzi, M., Siemiginowska, A., et al. 2009, A\&A, 501, 89

Tingay, S. J., Macquart, J.-P., Collier, J. D., et al. 2015, AJ, 149, 74

Tremblay, S. E., Taylor, G. B., Ortiz, A. A., et al. 2016, MNRAS, 459, 820

Ueda, Y., Eguchi, S., Terashima, Y., et al. 2007, ApJL, 664, L79

van Breugel, W., Miley, G., \& Heckman, T. 1984, AJ, 89, 5

Vink, J., Snellen, I., Mack, K.-H., \& Schilizzi, R. 2006, MNRAS, 367, 928

Yaqoob, T. 2012, MNRAS, 483, 3360

Younes, G., Porquet, D., Sabra, B., et al. 2010, A\&A, 517, A33

Young, M., Elvis, M., \& Risaliti, G. 2009, ApJS, 183, 17 